\title{
Distributed Dynamic Mobility Management Scheme for Network Mobility
}

\author{
Asif Ali Wagan ${ }^{1}$, Low Tang Jung ${ }^{2}$ \\ asifwaggan@gmail.com ${ }^{1}$,lowtanjung@utp.edu.my ${ }^{2}$ \\ Computer and Information Sciences Department \\ Universiti Teknologi PETRONAS \\ 32610 Seri Iskandar, Perak, Malaysia
}

\begin{abstract}
Various network management research communities have developed mobility management solutions for the Network Mobility Basic Support Protocol (NEMO-BSP) to allow seamless attachment between heterogeneous networks. The mobility management is the mobility solution dealing with a node's location update and its data delivery. NEMO is a host-based scheme that is still facing QoS issues. Whereas, the network-based mobility schemes have significantly reduce the involvement of the host in the node mobility tasks, but due to the centralized entity approach this scheme is considered less scalable. The Distributed Mobility Management (DMM) scheme is more scalable and aims to alleviate the traditional network mobility management schemes. In this paper, we propose a new DDMM scheme, which is aimed to reduce the tunneling cost and the overall packet delivery cost by implementing the Distributed Local Fix Routers (DLFRs). The results obtained demonstrate that the suggested DDMM scheme is outperformed other known schemes in terms of these two metrics.
\end{abstract}

Keywords: Network Mobility, Distributed Mobility Management, Tunnel Cost, Networkbased Mobility Management, Distributed Local Fix Router.

\section{Introduction}

Network Mobility (NEMO) is a special category of technology in Mobile Internet Protocol (MIPv6)[1]. In NEMO, a mobile router can be deployed in any transportation media such as buses, private vehicles, marine vessels, aircrafts etc. The mobile router is responsible for connecting itself to the mobile devices that are available in the transportation media. The mobile router shall get connected with the available internet service stations surrounding it such as Wifi, WiMax, UTMS, Hotspot and cellular base station, etc. The Network Mobility Basic Support Protocol (NEMO-BSP) was designed to provide seamless internet connections to all mobile nodes throughout the users' journey [1] while traveling in the moving vehicles. During this journey, the mobile router travels between one network environment to another network environment. In such situation, the mobile router is crucial to keep all active nodes in a consistent mobile connectivity.

The initial mobile management protocol was known as MIPv4 and MIPv6 [2], [3]. These protocols were weak in mobile connectivity due to two reasons. Primarily, the complex structure of these protocols was not suitable to support a high number of passive sensors and wireless devices to operate and to connect effectively to a network. Secondly, whenever a node is connected to the mobile router (on a mobile network), it needs to initiate a link-level hands- 
off in order to move from one network segment to another network segment. The MIPv4 and MIPv6 protocols are inefficient in this capacity.

Researchers have proposed various mobility management schemes. These mobility management schemes can be divided into three main groups i.e. the Host-based scheme, Network-based scheme, and Distributed Mobility Management (DMM) based scheme [4]. In this paper, we proposed a mobility management scheme based on DMM. DMM is widely used to mitigate scalability issues in the traditional mobility management approaches [5]. Our proposed mobility management approach is aimed to lessen packet delivery cost and tunnel cost in mobile node connectivity (during the point of attachment from one router to another router) in the network.

This research article is organized as follows. In section 2, the mobility management schemes and the gaps between the existing schemes are highlighted via literature review. Section 3 discusses the DMM overview and its types. Our mobility management approach is proposed in Section 4. Some results obtained from the proposed scheme are discussed in Section 5. Section 6 concludes this paper with remarks for future work.

\section{Literature Review}

Many NEMO researchers in industry and academia have been focusing on improving the mobility management scheme for Network Mobility (NEMO) in mobile networks. One major outcome from these researches is a new mobility mechanism in the network-based mobility solution schemes known as Proxy Mobility Internet Protocol version 6 (PMIPv6)[6]. PMIPv6 was developed by IETF. In PMIPv6, the network part deals and support mobility operation of mobile nodes without the nodes' involvement in signaling operations. The Mobile Access Gateways (MAG) is responsible for managing all mobility signaling operations. PMIPv6 scheme has two main entities: Mobile Access Gateway (MAG) and Local Mobility Anchor (LMA). These two entities handle all mobility management by sending proxy-binding updates. This MAG entity in PMIPv6 is use to record the movement of the nodes. When any node/host reach at the edge of the network segment subsequently the mobility entities initiates mobility signal operation for the node. This entity-based approach provides the non-involvement of the host when moving from one network segment to another. The PMIPv6 uses proxy mobility agent to deal the signal and movement operations on the behalf of the mobile nodes.

The traditional network (such as MIPv6-based) was designed to support individual mobile nodes and does not perform according the QoS requirement. The PMIPv6 protocol, on the other hand, can support either Mobile IP client nodes functionality or without that mobility function. Furthermore, in the [RFC 5213] specifications, PMIPv6 is backward compatible with IPv4 node, IPv6 node, or dual stack node.

In the network-based mobility management techniques, a central entity is used in the mobility management operations. It is considered the core (central) entity. The core manages the mobility management, inclusive of mobility routing, prefix allocation, and node location management. The NPMIPv6 in [7] is a network-based mobility solution for NEMO applications. In the NPMIPv6, the LMA manages mobility operations for all connected mobile nodes. LMA is also handling the packet routing from the source node to the destination node(s). The networkbased approaches significantly improves network performance in terms of signalling overhead. Unfortunately, this approach has some drawbacks like central entity failure, lack of efficient resource management and low scalability. 
In the 5th generation network that supports heterogeneous traffic from multiple devices like Internet of Things (IoT), it is foreseen that the traffic volume will be multifold. In such situation, the current centralized entity architecture will not be able to cope with this traffic intensity. In particular, when multiple devices are sending data simultaneously. To address this issue, the 3GPP1 group has developed a solution called Evolved Packet System (EPS) to decrease its involvement with the core entity [12] [13]. Subsequently, it is necessary to configure the network close to the user for providing better service experience. So, it will be possible to bypass a complex operators backhaul and core infrastructure. In this perspective, small size networks can provide better services because they can be implemented in transportation vehicles, canteen, or small malls.

To reduce the handover delay, the author in [8] suggested a partially distributed mobility management scheme. The design principle in this scheme was based on the PMIPv6 approach. However, the mobility management operations were distributed and a local access router performed the local mobility functions. A mobile router was connected to multiple network interfaces and handles all mobility functions. However, The qualitative results showed that this scheme can reduce the handover delay and packet delivery cost.

A full Distributed Mobility Management (DMM) approach was suggested in [9] called DNEMO. In this scheme, there was no central entity involvement in the mobility management operations. Consequently, all mobility management functions are transferred to the local access router. The D-NEMO eliminates the participation of central entity to bring along some improvements in terms of handover delay. However, due to complicated structure this scheme fails to provide effective packet delivery ratio.

\section{Distributed Mobility Management (DMM) Overview}

The traditional mobility management protocols for a mobile system are highly dependent on the intelligent centralized entity. There are two types of traditional mobility techniques: hostbased techniques and network-based techniques [10]. These techniques are divided into subcategories in mobility management such as MIPv6, dual stack MIPv6, and PMIPv6. However, both of these techniques are found to be ineffective in managing a large volume of mobile data traffic. This is due to the participation of the central entity (LMA in the PMIPv6 and HA in MIPv6) in all signal and routing operations for all attached mobile nodes. For instance, the home agent in the MIPv6 and the LMA in the PMIPv6 [12]. Other issues in regard to centralized mobility management are highlighted below from [11][12]. 
- Scalability: The traditional network is designed to support central management entity. All traffic is supposed to forward via this central entity. This creates the scalability and network design upgrading/enhancement issues due to the limited resources and routing capabilities in the centralized mode. Furthermore, the whole network system needs to equip beforehand with enough additional capabilities to cater for the unforeseen additional traffic loads.

- Reliability: As the current network architecture depends on the centralized entity, it is not uncommon that this central entity fails to provide effective responses due to enormous traffic volume or worse when the network is under malicious attack.

To mitigate these problems, the researchers have proposed new mobility management scheme known as Distributed Mobility Management (DDM). This scheme is to provide a flatter network architecture where the mobility entity is deployed as near to the user as possible. In addition, this DMM scheme also provides control and data units and install them at the edge of the network. Notably, DMM is a promising mechanism that supports mobile nodes (MNs) to roam around different mobile network units. This is not supported by the existing mobility management mechanisms. The commercial research industries and standardization group, IETF and 3GPP, are working on DDM with promising outcomes[13].The DMM schemes can be adopted in any traditional mobility management schemes such as routing-based DMM, PMIPv6-based DMM, and MIPv6-based DMM [8], [14]-[17]. Among these categories, it is noticed that PMIPv6-based DDM scheme is able to achieve good results as compared to the conventional PMIPv6 scheme under the heavy traffic situation.

Subsequently, the DMM scheme is itself divided into two main groups i.e. Partial-DMM and Fully-DMM. The Partial-DDM approach follows the footsteps of PMIPv6 and deployed the central entity in the network architecture [17]. Whereas in the Fully-DMM approach, it does not require any central entity. It should be noted here that the DMM approach is still under much exploration among the research communities. The Distributed Mobility Management (DMM) conceptual architecture is shown in Fig. 1[18]. DMM has enhanced access to internet protocol services and supports heterogeneous access network [4][8][9]. The DMM is an IP mobility solution, which adapts the existing IP mobility solutions of MIPv6 and PMIPv6, by evolving the flat IPv6 mobile network architecture. DMM can select optimal routes for data packets by deploying the multiple access points and establishing dense network area for users. This dense network area is to provide much better services to the users by simplifying the complex IP mobility network structure. 


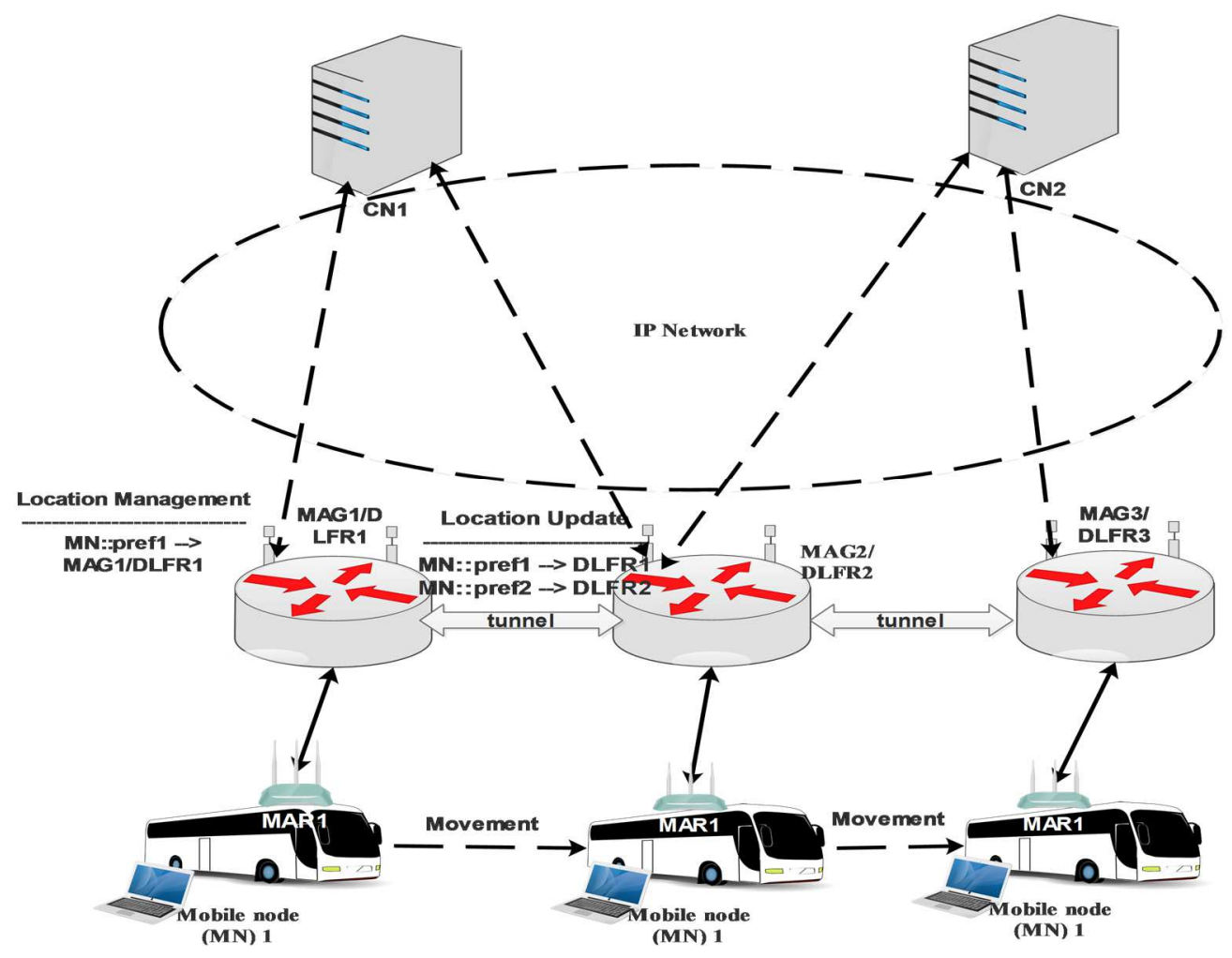

Fig. 1. Fully-Distributed Mobility Management

In DMM the point of attachment can migrate from one access router to another access router. The point of attachment events take place at Mobile Access Router (MAG) or DLFR level and the rest of the network are unaware about this activity (transparent attachment) as illustrated in Fig. 1[18]. Each MAG in DMM has a function to support this mobility operation. Furthermore, DMM is able to support dynamic mobility as when it is required. For example, when any MN is performing handoff in the DMM environment, only the MAG is to change for a new session. In comparison to existing protocols (MIPv6/PMIPv6) all handoff operations are managed by the core entity i.e. HA/LMA respectively. This new session in DMM is established on the MAG level and initiated by using the IPv6 address. Take note that the IPv6 has the feature to use multiple IP addresses at the same time. In this respect, whenever a data is sent on a new session it does not need any tunnel among the $\mathrm{MN}$ and the correspondent node $(\mathrm{CN})$ until the handover is performed completely. In the situation where $\mathrm{MN}$ is performing one or multiple IP handover without terminating the previous session, the data are sent via tunneling. It is reported in [10] that DMM has significantly improved the routing of data traffic. 
The DMM approach is to reduce the tunneling overhead as well as impacting the signal operation load. The DMM's mobility anchors are deployed topologically near to the host device to provide good network performance. In order to overcome the single point of failure issue, the binding message is carried out by MAG and not by the core entity such as that in the conventional centralized entity methods.

\section{The Proposed DMM Scheme}

This section describes the proposed DDMM scheme. Fig. 2 shows the proposed DDMM network architecture to support the proposed mobility scheme. This network architecture contains five main entities, namely Mobile Node (MN), Mobile Access Router (MAR), Distributed Local Fix Router (DLFR), Regional Access Router (RAR) and Correspondent Node $(\mathrm{CN})$. This proposed scheme is using multiple data links to fetch the data packets from different CNs by implementing the RAR. It does not contain any centralized entity. RAR is the solution to issues like scalability and single point failure.

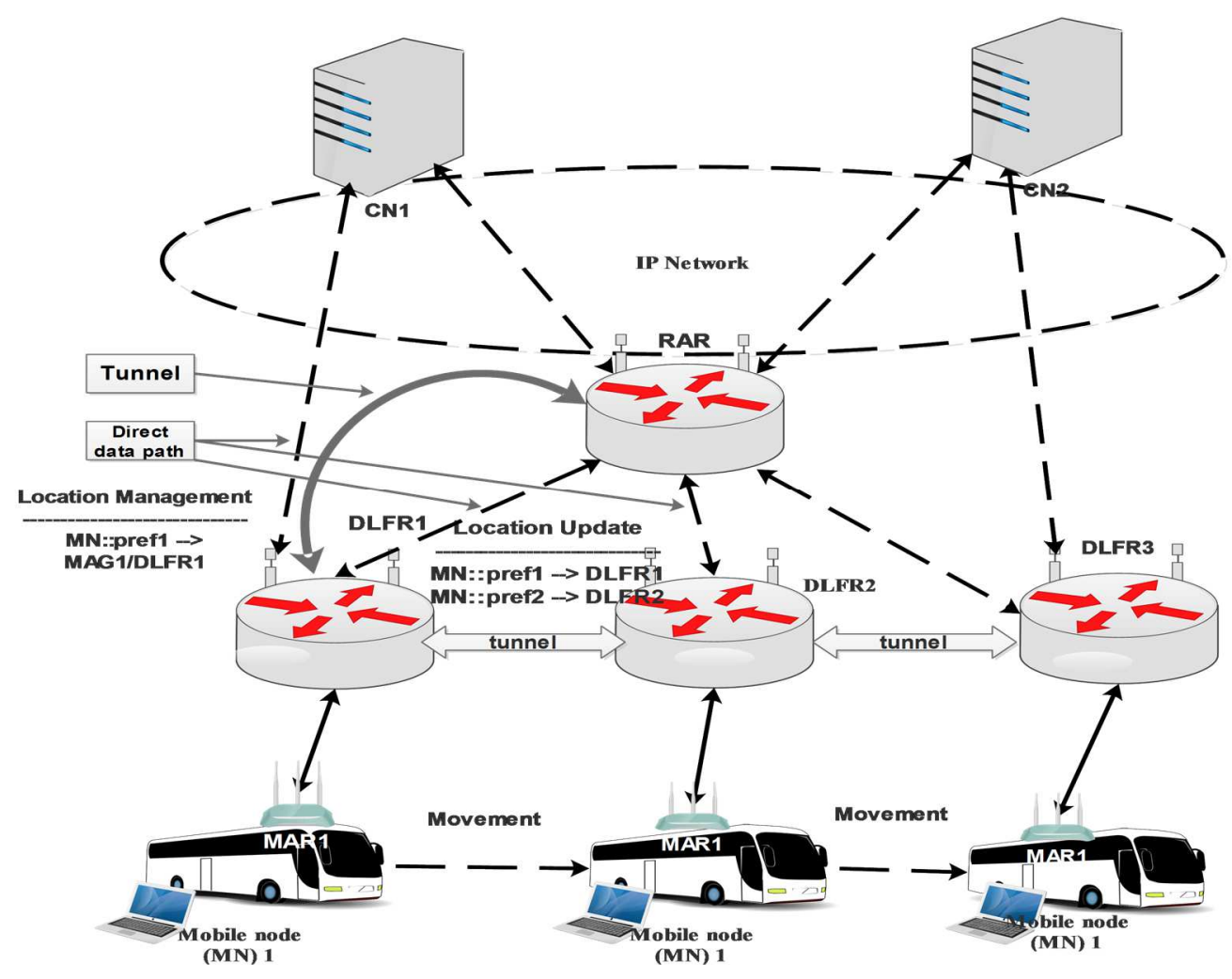

Fig 2. The proposed DMM Network Architecture 
The RAR is a hierarchical router, which supports more than one DLFRs and dynamically changes the data link whenever MAG is attached to new DLFR. RAR is responsible to establish a connection with next available DLFR. The RAR covers multiple DLFRs, shall establish tunnels between DLFR and MAR, whenever MAR1 is not in the signal coverage of a particular DLFR as illustrated in Fig 2, and described in Fig. 3. The DLFRs are responsible to keep tracking the MAR1 and updates the RAR accordingly.

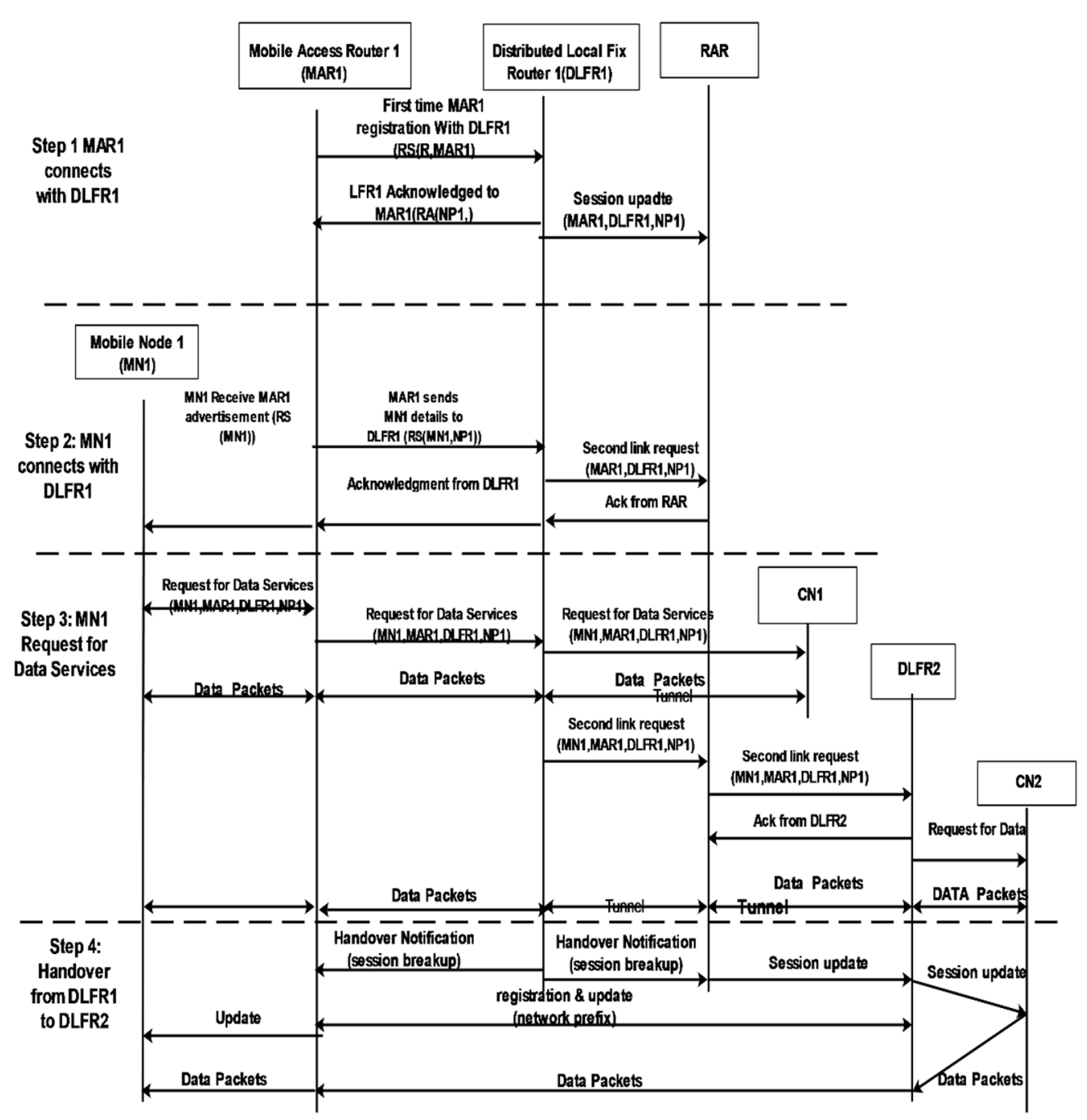

Fig 3. Proposed mobility management scheme based on DMM approach 
In the proposed scheme, router discovery involves different kinds of ICMP packet. The router advertisement message is sent periodically by the router or after receiving router solicitation message from the host. The router advertisement message comprises prefixes that are used to determine the current address, serving hops and configuration. The router solicitation message is sent by the host when it encounters to new interface. After receiving the message by current serving DLF router, the host replies back with router advertisement message. Flag pointers are used in our proposed scheme to differentiate between various types of messages. Basically, the flag pointer is placed in a message to indicate to the DLFRs regarding the binding update and the MN. In conjunction with the proposed DDMM scheme, the flags are used to differentiate between the router solicitation messages.

The proposed distributed dynamic mobility management scheme has 4 operation steps as shown in Fig 3. The first two steps describe the initial registration of mobile access router and mobile node with the network respectively. In Step 1, the registration between MAR1 and DLFR1 takes place. In this process, MAR1 sends router solicitation message that contains the MAR's ID and its location. The DLFR1 verify the MAR1 ID and the location, then it sends the IP Prefix address and IP address pool token to MNs. Furthermore, DLFR1 communicates with Regional Access Router (RAR) to store the MAR1 configuration.

In Step 2, MN1 attached with the MAR1, then MN1 sends MN's ID in response to the MAR1 router solicited (RS). MAR1 generates the IP address from IP address pool for MN1. In addition, MAR1 sends MN1's IP address and location details to the DLFR1. In response, DLFR1 sends an acknowledgment with a network prefix (NP). The DLFR1 updates the RAR regarding the MN1 configurations. Step 3 and Step 4 describe the data delivery mode and handoff procedure respectively. In Step 3 (Data Mode), when MN1 requests for data service from the MAR1, MAR1 sends a request to the DLFR1. The DLFR1 shall perform two operations: (1) DLFR1 first directly communicates with CN; and (2) at the same time the DLFR1 requests RAR for the second link. The RAR communicates with DLFR2 and receives the data packets. The tunnel is established between RAR and DLFR1 for receiving data packets. In Step 4 the handoff process is defined. When MAR1 reaches at the edge of the DLFR1 signal coverage, the DLFR1 triggers handoff process and sends an acknowledgment message to MAR1 and RAR. Subsequently, MAR1 shall enters into the DLFR2 coverage region. The RAR is responsible to update the DLFR2 for MAR1 ongoing sessions and maintain the connectivity. DLFR2 registers and update all the ongoing sessions. In addition, it removes the tunnel and sends data packets directly to MAG1.

\section{Results and Discussion}

The tunnel cost and the packet delivery cost are the two metrics discussed in this section. Results were compiled on the basis of analytical formulae adopted from the analysis model and network topology found in [4][9][19]. Fig 4 shows that all schemes manifest a linear increases of tunnel costs as number of MAGs increases. However, the proposed schemes show an overall reduction of tunnel cost. Whenever multiple DFRs are used it does impact the tunnel cost but still less as compared with other schemes. 


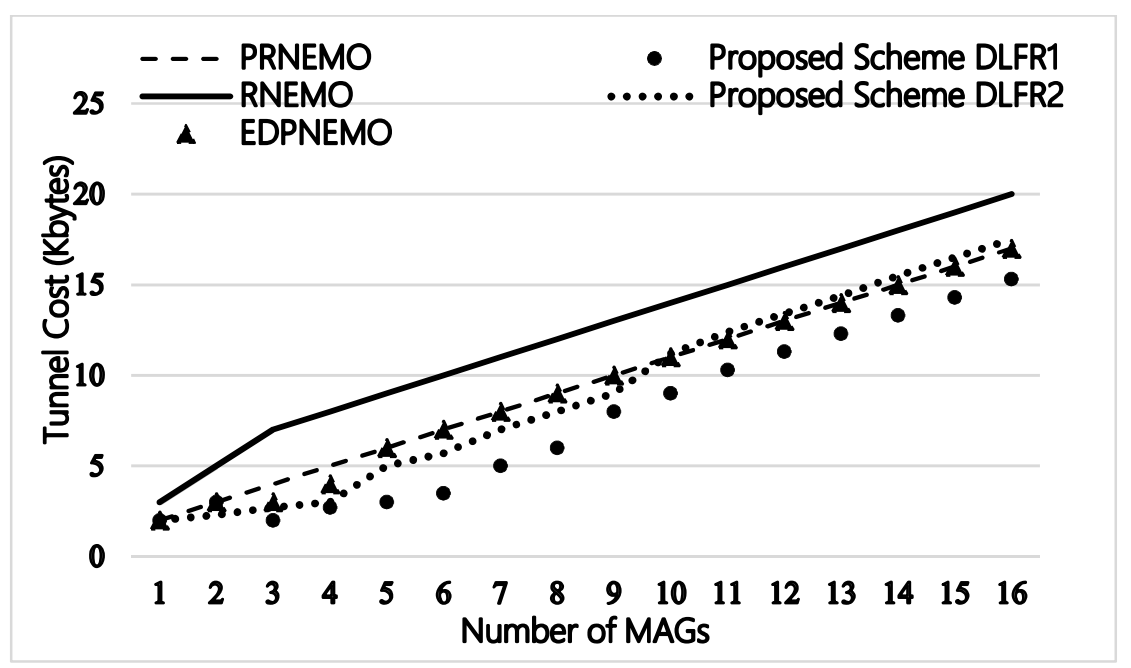

Fig. 4. Number of MAGs v/s Tunnel Cost

Comparison of packet delivery cost between the suggested scheme and other known schemes is shown in Fig. 5. It is noticeable that the suggested method has the lowest cost among all the schemes. This is mainly due to the reduction of the fetching data packet multiple sources and tunnel size.

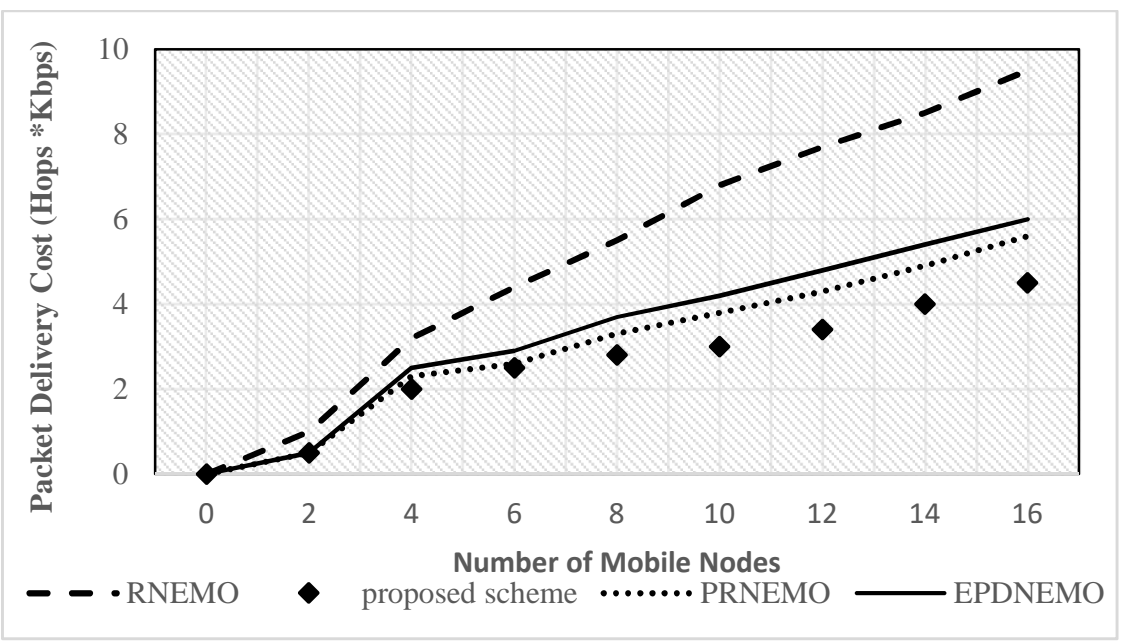

Fig. 5. Packet delivery cost v/s number of MNs 


\section{Conclusion and Future Work}

The Distributed Dynamic Mobility Management (DDMM) is a new flat architecture proposed for the mobility management in a mobile network environment. It has the capability to cope with many conventional schemes issues. The DDMM decouples locally the traffic in order to reduce unnecessary transactions. In this article, we have presented the mobility management scheme based on the DMM approach. The proposed scheme supports multiple data links to address the single point failure issue. The Regional Access Router (RAR) is proposed to dynamically change the data links from tunnel to direct link (for data packet delivery) whenever it is necessary. The dynamic changes in communication link and fetch data packets from different sources reduce the packet delivery cost. Two metrics (packet delivery cost and tunnel cost) were used to compare the proposed scheme against some commonly deployed schemes. The results obtained from the proposed scheme show better performance than the other schemes. In the future work, we will analyse the suggested method using other relevant metrics and benchmark against more similar schemes.

\section{References}

[1] V. Devarapalli, R. Wakikawa, A. Petrescu, and P. Thubert, "Network mobility (NEMO) basic support protocol," techreport, 2004.

[2] C. Perkins and others, "RFC 5944 IP Mobility Support for IPv4.” Revised, 2010.

[3] C. Perkins, D. Johnson, and J. Arkko, "RFC 6275: mobility support in IPv6," Internet Eng. Task Force, 2011.

[4] S. Jeon and Y. Kim, "Cost-efficient network mobility scheme over proxy mobile IPv6 network," IET Commun., vol. 5, no. 18, p. 2656, 2011.

[5 ] D.-H. Shin, D. Moses, M. Venkatachalam, and S. Bagchi, "Distributed mobility management for efficient video delivery over all-IP mobile networks: Competing approaches," Network, IEEE, vol. 27, no. 2, pp. 28-33, 2013.

[6] S. Gundavelli, K. Leung, V. Devarapalli, K. Chowdhury, and B. Patil, "Proxy mobile ipv6," techreport, 2008.

[7] I.Soto, C. J. Bernardos, M. Calderon, A. Banchs, and A. Azcorra, "NEMO-enabled localized mobility support for internet access in automotive scenarios," Commun. Mag. IEEE, vol. 47, no. 5, pp. 152-159, 2009.

[8] T.-X. Do and Y. Kim, "EPD-NEMO: efficient PMIPv6-based distributed network mobility management," Wirel. Networks, 2015.

[9] T.-X. Do and Y. Kim, "Distributed network mobility management," in Advanced Technologies for Communications (ATC), 2012 International Conference on, 2012, vol. 6, pp. 319-322.

[10] E. Perera, V. Sivaraman, and A. Seneviratne, "Survey on network mobility support," SIGMOBILE Mob. Comput. Commun. Rev., vol. 8, no. 2, pp. 7-19, 2004.

[11] H. Chan and others, "Problem statement for distributed and dynamic mobility management," Draft. (work progress), 2011.

[12] C. J. Bernardos, J. C. Zuiga, and A. Reznik, "Towards flat and distributed mobility management: A 3GPP evolved network design,” IEEE Int. Conf. Commun., pp. 6855-6861, 2012.

[13] J. C. Zúniga, C. J. Bernardos, A. de la Oliva, T. Melia, R. Costa, and A. Reznik, "Distributed mobility management: a standards landscape,” IEEE Commun. Mag., vol. 51Zúniga, no. 3, pp. 80-87, 2013.

[14] H. A. Chan, "Proxy mobile IP with distributed mobility anchors," 2010 IEEE Globecom Work., pp. 16-20, Dec. 2010. 
[15] P. Bertin, S. Bonjour, and J.-M. Bonnin, "Distributed or centralized mobility," in Global Telecommunications Conference, 2009. GLOBECOM 2009. IEEE, 2009, pp. 1-6.

[16] C. Bernardos and J.-C. Zúñiga, "PMIPv6-based distributed anchoring," 2015.

[17] F. Giust, A. La Oliva, and C. Bernardos, "Flat access and mobility architecture: An IPv6 distributed client mobility management solution," in Computer Communications Workshops (INFOCOM WKSHPS), 2011 IEEE Conference on, 2011, pp. 361-366.

[18] H. Yokota, P. Seite, E. Demaria, and Z. Cao, "Use case scenarios for distributed mobility management," IETF Draft. Nov, 2010.

[19] C. Park, J. Park, H. Wang, and H. Choo, "Efficient network mobility support scheme for proxy mobile IPv6,” EURASIP J. Wirel. Commun. Netw., vol. 2015, no. 1, p. 210, 2015. 